\title{
Vitamin D supplementation for primary dysmenorrhea: a double-blind, randomized, placebo-controlled trial
}

\author{
Fatemeh Alsadat Rahnemaei, MSc1 , Ali Gholamrezaei, MD, PhD², Maryam Afrakhteh, MD³ , Farid Zayeri, $\mathrm{PhD}^{4}$, \\ Mohammad Reza Vafa, $\mathrm{PhD}^{5}$, Arian Rashidi, MD ${ }^{6}$, Giti Ozgoli, PhD ${ }^{1}$

\begin{abstract}
'Department of Midwifery and Reproductive Health, School of Nursing and Midwifery, Midwifery and Reproductive Health Research Center, Shahid Beheshti University of Medical Sciences, Tehran, Iran, ${ }^{2}$ Pain Management Research Institute, Faculty of Medicine and Health, The University of Research Center and Department of Biostatics, Faculty of Paramedical Sciences, Shahid Beheshti University of Medical Sciences, ${ }^{5}$ Department of Nutrition, School of Public Health, Iran University of Medical Sciences, ${ }^{6}$ Clinical Pathology Laboratory, Shohadae Tajrish Hospital, Shahid Beheshti University of Medical Sciences, Tehran, Iran
\end{abstract} \\ Sydney, Sydney, Australia, ${ }^{3}$ Department of Obstetrics and Gynecology, Shohada Hospital, Shahid Beheshti University of Medical Sciences, ${ }^{4}$ Proteomics
}

\section{Objective}

Recent studies have shown a possible association between vitamin D deficiency and the severity of primary dysmenorrhea. The present study aimed to investigate the effect of vitamin D supplementation on pain and systemic symptoms in patients with primary dysmenorrhea.

\section{Methods}

This double-blind, randomized, placebo-controlled trial was conducted on female students aged 18 to 32 years with primary dysmenorrhea and vitamin D deficiency $(25[\mathrm{OH}] \mathrm{D}<30 \mathrm{ng} / \mathrm{mL})$. The participants $(n=116)$ received either $50,000 \mathrm{IU}$ of vitamin D3 (cholecalciferol) or placebo capsules on a weekly basis for eight consecutive weeks. The outcomes were pain intensity (scored 0 to 10), number of days with pain, number of consumed pain-relief medications (per day), and severity of systemic symptoms (fatigue, headache, nausea/vomiting, and diarrhea; total score of 0 to 12).

\section{Results}

Compared with baseline, our participants who received vitamin $D$ experienced significant reductions in pain intensity $(-1.0$ and -1.5 score at weeks 4 and $8, P<0.001)$, the number of days with pain $(-1.0$ day at weeks 4 and $8, P<0.001)$, the number of consumed pain-relief medications $(-1.0$ at weeks 4 and $8, P<0.001)$, and systemic symptoms severity $(-1.0$ score at weeks 4 and $8, P<0.001)$. No significant improvements were observed in the placebo group in terms of these outcomes.

\section{Conclusion}

Vitamin D supplementation in women with primary dysmenorrhea and vitamin D deficiency could improve systemic symptoms and reduce pain intensity, the number of days with pain, and the need for consuming pain-relief medications.

Keywords: Dysmenorrhea; Vitamin D; Cholecalciferol; Pain

\section{Introduction}

Primary dysmenorrhea is defined as menstrual pain in the absence of pelvic pathology and is the most common menstrual symptom among adolescent girls and young women [1]. Pain usually occurs just before or at the beginning of menstruation and lasts for 8-72 hours [2]. Headache, fatigue, nausea/vomiting, and diarrhea during menstruation are also common primary dysmenorrhoea-associated systemic symptoms [3]. The severity of dysmenorrhea usually improves after childbirth [4]. The prevalence of dysmenorrhea in women of reproductive age is $16 \%$ to $91 \%$ [5]. In a systematic review
Received: 2020.10.21. Revised: 2021.02.16. Accepted: 2021.04.26. Corresponding author: Giti Ozgoli, PhD

Department of Midwifery and Reproductive Health, School of Nursing and Midwifery, Midwifery and Reproductive Health Research Center, Shahid Beheshti University of Medical Sciences, Niayesh Intersection, Vali Asr Street, Tehran 1985717443, Iran E-mail: gozgoli@gmail.com

https://orcid.org/0000-0003-2111-7024

Articles published in Obstet Gynecol Sci are open-access, distributed under the terms of the Creative Commons Attribution Non-Commercial License (http://creativecommons. org/licenses/by-nc/3.0/) which permits unrestricted non-commercial use, distribution, and reproduction in any medium, provided the original work is properly cited.

Copyright $\odot 2021$ Korean Society of Obstetrics and Gynecology 


\title{
Obstetrics \& Gynecology Science
}

\author{
Vol. 64, No. 4, 2021
}

and meta-analysis conducted in Iran, the overall prevalence of primary dysmenorrhea was estimated to be $71 \%$ [6]. Dysmenorrhea has negative impacts on daily activities and causes absenteeism from school, university classes, and work. It is also responsible for the significant loss of earnings and diminished quality of life $[7,8]$. Moreover, dysmenorrhea could create a feeling of fear from the onset of the next menstrual cycle, compromising the mental health of women between menstruation periods. In addition to economic burdens, dysmenorrhea can also affect women's social and familial relationships [9].

A suggested mechanism causing primary dysmenorrhea is the increasing production of prostaglandins in the endometrium. Prostaglandins are derived from the arachidonic acid produced in cyclooxygenase and lipoxygenase pathways. The rise in the synthesis of prostaglandins in women with primary dysmenorrhea increases uterine tone and high-amplitude contractions. Upregulation of cyclooxygenase activity and prostaglandin synthesis are observed in women with dysmenorrhea [10]. Accordingly, nonsteroidal anti-inflammatory drugs (NSAIDs), which inhibit the expression of cyclooxygenase enzyme involved in the synthesis of prostaglandin, are used as the first-line treatment of primary dysmenorrhea [11]. These drugs are effective in managing primary dysmenorrhea; however, treatment with NSAIDs is also associated with several side effects, such as stomach pain and heartburn, increasing the risk of stomach ulcer and gastrointestinal bleeding $[12,13]$. Therefore, selection of other therapeutic options (e.g.,complementary) would perhaps allow women with primary dysmenorrhea to limit the use of NSAIDs and, thus, reduce the associated side effects.

The role of micronutrient deficiencies in the pathogenesis of primary dysmenorrhea has recently been highlighted. Decrease in the levels of micronutrients, for instance, betacarotene, vitamin $E$, and zinc, could increase the process of oxidation and production of more prostaglandins [14]. Intake of calcium may reduce the severity of primary dysmenorrhea by decreasing prostaglandin synthesis [15]. Recent studies have shown that the serum levels of vitamin $D$ reduce in the luteal phase of the menstrual cycle [16]. The role of vitamin $D$ deficiency in inducing or exacerbating the symptoms of primary dysmenorrhea has been addressed by some studies [17]. Vitamin D metabolites (e.g., 25[OH]D and 1,25[OH]2D) reduce the levels of inflammatory cytokines and associated contractile factors (prostaglandin) in the endometrium and incapacitate its biological activity by affecting its receptors in the endometrium $[18,19]$.

A few studies examined the effect of vitamin $D$ supplementation on primary dysmenorrhea symptoms and showed promising results $[20,21]$. However, only a few double-blind, randomized, placebo-controlled trials have been conducted in this regard [22-24]. Vitamin D deficiency has emerged as a global problem in the recent years. The prevalence of vitamin D deficiency in Iran, for example, is $64 \%$ in women [25]. Considering the possible role of vitamin D deficiency in exacerbating the severity of primary dysmenorrhea symptoms and given the promising findings of previous studies, the present study aimed to evaluate the effect of vitamin D supplementation on pain and systemic symptoms in women with primary dysmenorrhea who had vitamin D deficiency.

\section{Materials and methods}

\section{Study design and participants}

This study was a double-blind, randomized, placebocontrolled clinical trial. Necessary permissions were obtained from the Ethics Committee of the Faculty of Nursing and Midwifery at Shahid Beheshti University of Medical Sciences (IR.SBMU.PHNM.1396.772). The trial was registered at the Iranian Registry of Clinical Trials (code no. IRCT201707223860N32). Written consent was obtained from the participants after explaining the study protocol. A researcher visited four university dormitories and explained the study to all the students who were inclined to hear the descriptions. Students who had symptoms of primary dysmenorrhea in their last six menstrual cycles were invited to participate in the study, and those who were willing to participate were screened based on the following study inclusion criteria: aged 18 to 32 years (according to the age range of students living in the studied university dormitories), with regular menstruation cycles (22-35 days), with a pain score of $\geq 4$ on the 11-point numerical rating scale (NRS), and with vitamin $D$ deficiency defined as serum 25 -hydroxyvitamin $D$ (25[OH]D) level of $<30 \mathrm{ng} / \mathrm{mL}$ ( $75 \mathrm{nmol} /$ liter) according to the Endocrine Society clinical practice guideline [26]. Moreover, those with a history of pregnancy, with uterine abnormalities (e.g., fibroids, polyps, endometrial hypertrophy, and endometriosis) or ovarian disorders (e.g., ovarian cysts and polycystic ovary) detected in recent ultrasound examinations, 


\section{Obstetrics \& Gynecology Science}

Fatemeh Alsadat Rahnemaei, et al. Vitamin D for primary dysmenorrhea

who self-reported abnormal vaginal and cervical discharges, who self-reported kidney or liver diseases, who experienced major stressful life events (e.g., death of relatives) or surgery in the last six months, who engaged in smoking or alcohol drinking, who used hormonal contraceptive methods, who took calcium and/or vitamin D supplements in the past three months, and with known hypersensitivity to vitamin D supplements were excluded from the trial.

\section{Sample size considerations}

The required sample size was 58 participants per group, considering an $80 \%$ power for detecting at least one score difference between the two groups in terms of reduction in pain severity in the 11-point NRS, 10\% drop-out rate, and a two-tailed $P$-value of 0.05 . The sampling process is shown in Fig. 1.

\section{Randomization and blinding}

Using a permuted block randomization method with a block size of 4 [27], an independent statistician generated a random list from therapeutic allocations with an equal number of samples in the vitamin $D$ group and placebo group ( $n=58$ per group). A researcher, who was not involved in recruiting the participants or allocating them to the study groups, placed the drugs in two separate packages of identical ap- pearance bearing $A$ and $B$ codes. Vitamin $D$ and placebo capsules were identical in shape, size, and color. The second researcher, who recruited the participants and allocated them to the study groups based on the random list as well as the outcome assessor, was not aware of the drug codes.

\section{Interventions}

The participants who were allocated to the vitamin $D$ group received oral vitamin D3 capsules (50,000 IU cholecalciferol; Zahravi Pharmaceutical Company, Tabriz, Iran), while those who were allocated to the placebo group received identical placebo capsules (containing inert oil like corn, Zahravi Pharmaceutical Company, Tabriz, Iran) on a weekly basis for eight consecutive weeks, according to the Endocrine Society clinical practice guideline [26]. Compliance was assessed by asking the participants to complete a weekly drug consumption form; reminders were sent via text messages. Our participants were allowed to use NSAIDs on demands, and the number of pain-relief medications used (per day) was recorded.

\section{Assessments}

Pain intensity was measured using an 11-point NRS, with 0 indicating no pain, 5 moderate pain, and 10 the worst possible pain. The duration of pain was assessed based on the

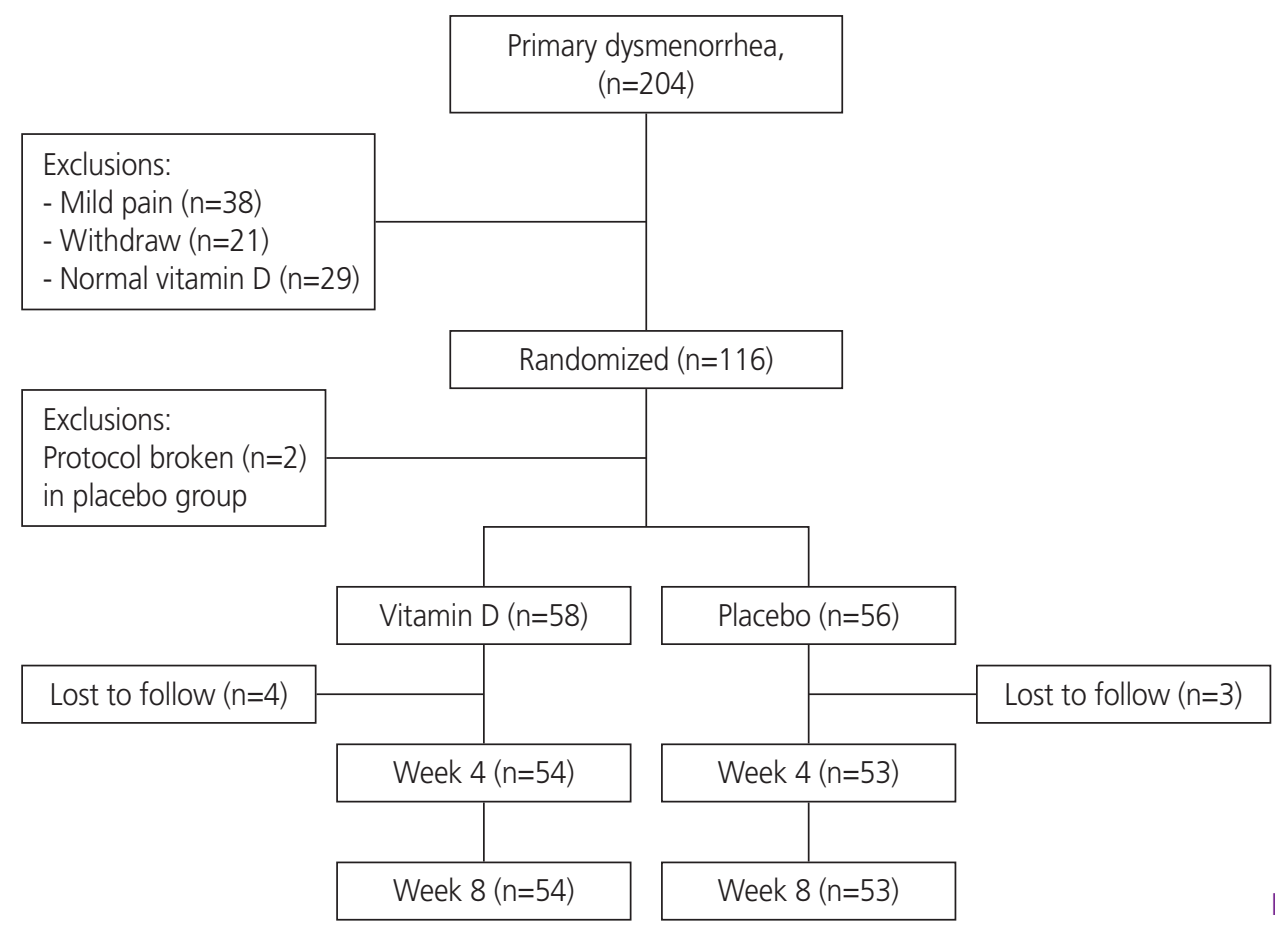

Fig. 1. Study flow diagram. 


\title{
Obstetrics \& Gynecology Science
}

\author{
Vol. 64, No. 4, 2021
}

number of days with pain in each menstrual cycle. Systemic symptoms included fatigue, headache, nausea/vomiting, and diarrhea and were assessed using a 3-point Likert scale with the following descriptions: 0 (none), 1 (mild, no need for medication), 2 (moderate, affecting daily activities and sometimes requiring medication), or 3 (severe, discontinuation of daily activities and absence of satisfactory response to medication). The total score for systemic symptoms could range from 0 to 12 . Moreover, the participants were asked to indicate the number of consumed pain-relief medications per day during the menstrual cycle. These self-reported study outcomes were assessed at baseline and then at weeks 4 and 8 from the beginning of the study.

The participants' blood samples were obtained during the luteal phase of the menstrual cycle (based on the last menstrual period), transferred to the refrigerator in a cold box, kept at 2-8 degrees centigrade, and transported to the laboratory the next morning. Serum levels of $25(\mathrm{OH}) \mathrm{D}$ were measured at baseline and then at week 8 using the chemiluminescence method (LIAISON ${ }^{\circledR}$ analyzer; DiaSorin, Saluggia, Italy). All the tests were conducted in the laboratories of university-affiliated hospitals with an intra-rater agreement of $>80 \%$.

The side effects were assessed at week 8 using a separate questionnaire and grouped in the following categories: gastrointestinal symptoms (nausea, vomiting, diarrhea, and dyspepsia), hypersensitivity (fever, urticaria, respiratory problem, itching, and rash), menstrual bleeding (same, increased), and others (tinnitus, headache, and dizziness). All questions were answered with yes or no.

\section{Statistical analyses}

The primary outcome of this study was pain intensity. The secondary outcomes included the number of days with pain, systemic symptoms severity, and the number of pain-relief medications used. We hypothesized that supplementation with vitamin D would reduce the intensity of pain, the number of days with pain, use of pain-relief medications, and the severity of systemic symptoms compared with placebo. The obtained data were analyzed utilizing JASP (version 0.14.1, University of Amsterdam). The data were not normally distributed according to the Shapiro-Wilk test. Therefore, the variables were compared between and within the groups using non-parametric tests (Mann-Whitney $U$ and Friedman tests, respectively). After the Friedman test, post-hoc analy- ses were conducted using the Wilcoxon signed-rank test. The Hodges-Lejman Estimate was reported as an alternative effect size measure [28], and 95\% confidence intervals were reported where possible. $P$-value correction was performed for multiple comparisons using the Holm-Bonferroni method [29]. The mentioned $P$-values in the results were corrected, and $P$-values of $<0.05$ were considered significant in all analyses.

\section{Results}

\section{Recruitments}

After conducting a preliminary survey of 1,100 students, 204 with primary dysmenorrhea were invited to participate in the study. Thirty-eight students with mild dysmenorrhea (pain score of $<4$ ) were excluded, and 21 participants withdrew from the study before randomization. Afterward, 145 students with a pain score of $\geq 4$ were subjected to blood sampling in order to measure the serum vitamin $D$ levels; of them, 29 had normal levels ( $\geq 30 \mathrm{ng} / \mathrm{mL}$ ) and were therefore excluded. Eventually, 116 participants with vitamin

Table 1. Comparison of demographic and baseline data between the study groups

\begin{tabular}{lccc}
\hline & $\begin{array}{c}\text { Vit D } \\
(\mathbf{n}=54)\end{array}$ & $\begin{array}{c}\text { Placebo } \\
(\mathbf{n}=53)\end{array}$ & $\boldsymbol{P}^{\text {-value }}{ }^{\text {a) }}$ \\
\hline Age $(\mathrm{yr})$ & $22.7 \pm 3.9$ & $22.5 \pm 3.3$ & 0.895 \\
BMl $\left(\mathrm{kg} / \mathrm{m}^{2}\right)$ & $24.0 \pm 2.6$ & $23.3 \pm 3.1$ & 0.273 \\
Age at menarche (yr) & $14.0 \pm 1.2$ & $14.0 \pm 1.5$ & 0.490 \\
Menstrual cycle interval (day) & $23.2 \pm 2.9$ & $23.9 \pm 3.3$ & 0.286 \\
Duration of bleeding (day) & $6(5,7)$ & $5(5,7)$ & 0.245 \\
Serum 25[OH]D level (ng/mL) & $20.0 \pm 6.0$ & $19.5 \pm 5.5$ & 0.715 \\
Pain intensity & $7.0 \pm 1.7$ & $6.6 \pm 1.5$ & 0.392 \\
Days with pain & $2(1,3)$ & $2(2,3)$ & 0.193 \\
Pain medication (day) & $2(1,3)$ & $1(0,2)$ & $0.042^{b)}$ \\
Nausea/vomiting & $2(1,3)$ & $2(1,3)$ & $>0.999$ \\
Diarrhea & $0(0,1)$ & $0(0,1)$ & $>0.999$ \\
Headache & $1(0.2,2)$ & $1(1,2)$ & 0.636 \\
Fatigue & $2(1,3)$ & $2(1,3)$ & 0.447 \\
Systemic symptoms total score & $5.5 \pm 2.3$ & $5.2 \pm 2.6$ & 0.485
\end{tabular}

Values are presented as mean \pm standard deviation or median (interquartile range $25 \%$, $75 \%$ ).

$\mathrm{BMI}$, body mass index.

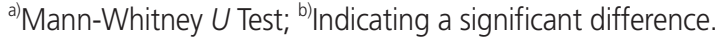




\section{Obstetrics \& Gynecology Science}

Fatemeh Alsadat Rahnemaei, et al. Vitamin D for primary dysmenorrhea

D levels of $<30 \mathrm{ng} / \mathrm{mL}$ were enrolled and randomized into two groups ( $n=58$ per group). Two participants in the placebo group who started the treatment for vitamin $D$ deficiency were excluded prior to receiving the intervention. Seven participants (four in the vitamin D group and three in the placebo group) who left the dormitory were excluded from the study. Finally, the data of 54 participants in the vitamin D group and those of 53 participants in the placebo group were analyzed (Fig. 1).

\section{Comparison of baseline characteristics between the two groups}

No differences were observed between the two groups in terms of demographic characteristics and menstrual status, or baseline measures; however, the vitamin D group had higher rate of pain-relief medication use than the placebo group (Table 1).

\section{Effect of the intervention on vitamin D status}

The serum level of $25(\mathrm{OH}) \mathrm{D}$ significantly increased from $20.0 \pm 6.0 \mathrm{ng} / \mathrm{mL}$ to $37.2 \pm 9.4 \mathrm{ng} / \mathrm{mL}$ in the vitamin $D$ group $(P<0.001)$, yet no significant changes were observed in the placebo group (from $19.5 \pm 5.5 \mathrm{ng} / \mathrm{mL}$ to $19.7 \pm 5.6 \mathrm{ng} / \mathrm{mL}$, $P=0.057)$. Of note, $13(24.0 \%)$ of the participants receiving vitamin D supplements still showed low levels of $25(\mathrm{OH}) \mathrm{D}$ $(<30 \mathrm{ng} / \mathrm{mL})$ at week 8 .

\section{Effect of the intervention on pain-related outcomes}

The Friedman test showed a significant change in pain intensity in the vitamin $D$ group $(P<0.001)$, but no significant changes were found in the placebo group $(P=0.670)$. Post-hoc analyses revealed a significant reduction in pain intensity in the vitamin $D$ group at week $4(P<0.001)$ and week $8(P<0.001)$, as depicted in Table 2 . Moreover, the process of reducing pain intensity in the intervention and placebo groups within 8 weeks is shown in Fig. 2 .

There was a significant change in the number of days with pain in the vitamin $D$ group $(P<0.001)$, but no significant changes were observed in the placebo group $(P=0.905)$. As presented in Table 2, the post-hoc analyses illustrated a significant reduction in the number of days with pain in the vitamin $D$ group at week $4(P<0.001)$ and at week $8(P<0.001)$.

A significant change was observed in the number of painrelief medications used per day in the vitamin $D$ group $(P<0.001)$, but no significant changes were observed in the placebo group $(P=0.920)$. Post-hoc analyses showed a significant reduction in the number of pain-relief medications used per day in the vitamin $D$ group at week $4(P<0.001)$ and

Table 2. Comparison of the pain-related outcomes between and within the vitamin $D(n=54)$ and placebo ( $n=53)$ groups from baseline to week 8 after the intervention

\begin{tabular}{|c|c|c|c|c|c|}
\hline & Baseline & Week 4 & Week 8 & $P$-value $1^{\text {a) }}$ & $P$-value $2^{\text {b) }}$ \\
\hline \multicolumn{6}{|l|}{ Pain intensity } \\
\hline Vit D & $7.0 \pm 1.7$ & $6.3 \pm 1.7$ & $5.6 \pm 1.7$ & $<0.001^{c)}$ & $<0.001^{c)}$ \\
\hline Placebo & $6.6 \pm 1.5$ & $6.6 \pm 1.5$ & $6.5 \pm 1.6$ & $>0.999$ & 0.809 \\
\hline$P$-value $3^{\mathrm{d})}$ & 0.392 & 0.618 & 0.033 & & \\
\hline \multicolumn{6}{|c|}{ Days with pain } \\
\hline Vit D & $2(1,3)$ & $2(1,3)$ & $1(1,2)$ & $<0.001^{c)}$ & $<0.001^{c)}$ \\
\hline Placebo & $2(2,3)$ & $2(2,3)$ & $2(2,3)$ & $>0.999$ & $>0.999$ \\
\hline$P$-value $3^{\text {d) }}$ & 0.193 & 0.030 & $<0.001$ & & \\
\hline \multicolumn{6}{|c|}{ Pain medication (day) } \\
\hline Vit D & $2(1,3)$ & $1.5(0,2)$ & $1(0,2)$ & $<0.001^{c)}$ & $<0.001^{c)}$ \\
\hline Placebo & $1(0,2)$ & $1(0,2)$ & $1(0,2)$ & $>0.999$ & 0.803 \\
\hline$P$-value $3^{\mathrm{d})}$ & 0.042 & 0.546 & 0.441 & & \\
\hline
\end{tabular}

Values are presented as mean \pm standard deviation or median (interquartile range $25 \%, 75 \%$ ). $0=$ no symptom, $1=$ mild, $2=$ moderate, and $3=$ severe symptom.

${ }^{a}$ Wilcoxon signed-rank test, week 4 vs. baseline, corrected based on the Holm-Bonferroni method; b'Wilcoxon signed-rank test, week 8 vs. baseline, corrected based on the Holm-Bonferroni method; 'Indicating a significant difference; ${ }^{a}$ Mann-Whitney $U$ test, corrected based on the Holm-Bonferroni method. 


\section{Obstetrics \& Gynecology Science}

Vol. 64, No. 4, 2021

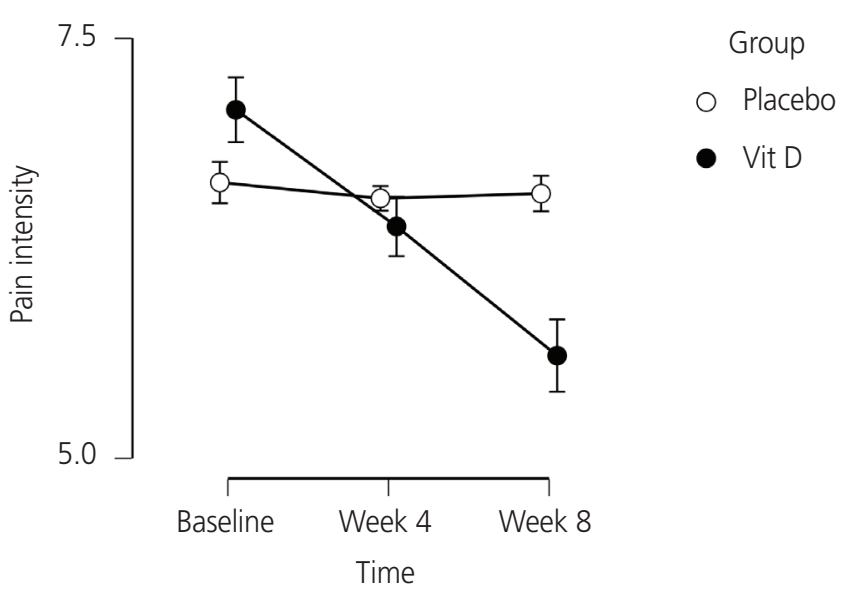

Fig. 2. Pain intensity over the study period in the vitamin $D$ and placebo groups. week $8(P<0.001)$ (Table 2$)$.

\section{Effect of the intervention on systemic symptoms}

The Friedman test indicated a significant change in the total score of systemic symptoms in the vitamin $D$ group $(P<0.001)$, but no significant changes were observed in the placebo group $(P=0.264)$. As represented in Table 3, posthoc analyses showed a significant reduction in the total score for systemic symptoms at week $4(P<0.001)$ in the vitamin $D$ group. With regard to the systemic symptoms, the Friedman test presented a significant change in the severity of headache $(P<0.001)$ and diarrhea $(P=0.006)$ in the vitamin $D$ group. Meanwhile, no significant changes were observed in the severity of headache $(P=0.368)$, fatigue $(P=0.368)$, nausea/vomiting $(P=0.607)$, or diarrhea $(P=0.449)$ in the pla-

Table 3. Comparison of systemic symptoms between and within the vitamin $D(n=54)$ and placebo $(n=53)$ groups from baseline to week 8 after the intervention

\begin{tabular}{|c|c|c|c|c|c|}
\hline & Baseline & Week 4 & Week 8 & $P$-value $1^{\text {a) }}$ & $P$-value $2^{\text {b) }}$ \\
\hline \multicolumn{6}{|c|}{ Nausea/vomiting } \\
\hline Vit D & $2(1,3)$ & $2(1,3)$ & $2(1,2)$ & 0.773 & $<0.001^{c)}$ \\
\hline Placebo & $2(1,3)$ & $2(1,3)$ & $2(1,3)$ & $>0.999$ & $>0.999$ \\
\hline$P$-value $3^{d)}$ & $>0.999$ & 0.761 & $>0.999$ & & \\
\hline \multicolumn{6}{|l|}{ Diarrhea } \\
\hline Vit D & $0(0,1)$ & $0(0,1)$ & $0(0,1)$ & $>0.999$ & $0.040^{c)}$ \\
\hline Placebo & $0(0,1)$ & $0(0,1)$ & $0(0,1)$ & 0.692 & $>0.999$ \\
\hline$P$-value $3^{d)}$ & $>0.999$ & 0.975 & 0.858 & & \\
\hline \multicolumn{6}{|l|}{ Headache } \\
\hline Vit D & $1(0.2,2)$ & $1(0,2)$ & $1(0,2)$ & $0.037^{c)}$ & $<0.001^{c)}$ \\
\hline Placebo & $1(1,2)$ & $1(1,2)$ & $1(1,2)$ & $>0.999$ & $>0.999$ \\
\hline$P$-value $3^{\mathrm{d})}$ & 0.636 & 0.730 & 0.180 & & \\
\hline \multicolumn{6}{|l|}{ Fatigue } \\
\hline Vit D & $2(1,3)$ & $2(1,3)$ & $2(1,2)$ & $0.026^{c)}$ & $0.002^{c)}$ \\
\hline Placebo & $2(1,3)$ & $2(1,3)$ & $2(1,3)$ & $>0.999$ & $>0.999$ \\
\hline$P$-value $3^{\text {d) }}$ & 0.447 & 0.72 & 0.884 & & \\
\hline \multicolumn{6}{|l|}{ Total score } \\
\hline Vit D & $5.5 \pm 2.3$ & $5.3 \pm 2.3$ & $4.7 \pm 2.2$ & $0.01^{c)}$ & $<0.001^{c)}$ \\
\hline Placebo & $5.2 \pm 2.6$ & $5.1 \pm 2.6$ & $5.2 \pm 2.5$ & 0.848 & 0.484 \\
\hline$P$-value $3^{\text {d) }}$ & 0.485 & 0.531 & 0.451 & & \\
\hline
\end{tabular}

Values are presented as mean \pm standard deviation or median (interquartile range $25 \%, 75 \%$ ). $0=$ no symptom, 1=mild, $2=$ moderate, and $3=$ severe symptom.

a) Wilcoxon signed-rank test, week 4 vs. baseline, corrected based on the Holm-Bonferroni method; b)Wilcoxon signed-rank test, week 8 vs. baseline, corrected based on the Holm-Bonferroni method; 'Indicating a statistically significant difference; ${ }^{\text {d) }}$ Mann-Whitney $U$ test, corrected based on the Holm-Bonferroni method. 


\section{Obstetrics \& Gynecology Science}

Fatemeh Alsadat Rahnemaei, et al. Vitamin D for primary dysmenorrhea

cebo group. Post-hoc analyses showed that at week 4, a significant reduction was observed in the severity of headache $(P=0.037)$ and fatigue $(P=0.026)$ in the vitamin $D$ group. At week 8 , a significant reduction was observed in the severity of headache $(P<0.001)$, fatigue $(P<0.001)$, nausea/vomiting $(P<0.001)$, and diarrhea $(P=0.020)$ in the vitamin $D$ group (Table 3, Fig. 3).

\section{Ancillary analyses}

As a secondary analysis, we assessed whether the amount of increase in vitamin D level was associated with the improvement in pain intensity in the vitamin D group. However, we found no significant correlations between these two variables $(P=0.166)$. Furthermore, no significant difference was observed between those who achieved a normal vitamin $D$ level ( $\geq 30 \mathrm{ng} / \mathrm{mL}$ ) after taking vitamin $\mathrm{D}$ supplements and those who did not achieve normal levels after receiving vitamin $D$ supplements in terms of pain intensity at week $8(P=0.572)$.

\section{Compliance and side effects}

The participants fully complied with the drug treatment and reported no specific side effects after taking vitamin D supplements or placebo at the end of the study.

\section{Discussion}

The results of this study revealed that treatment with vitamin D supplements in women with primary dysmenorrhea who have vitamin $D$ deficiency cannot only reduce the severity and frequency of pain, but could also decrease the severity of systemic symptoms and the need for pain-relief medications (such as NSAIDs).

Recently, two studies have shown the relationship between inadequate calcium intake and low levels of vitamin $D$ in adults with primary dysmenorrhea [30,31]. Calcium homeostasis is regulated by calcitonin, parathyroid hormone, and 25-hydroxyvitamin D [32]. Similar to the physiological effect of calcium on muscle contraction and rest, it could be expected that these hormones would also play a role in the pathogenesis of primary dysmenorrhea. Calcium can control smooth muscle contraction, tone, and relaxation, and
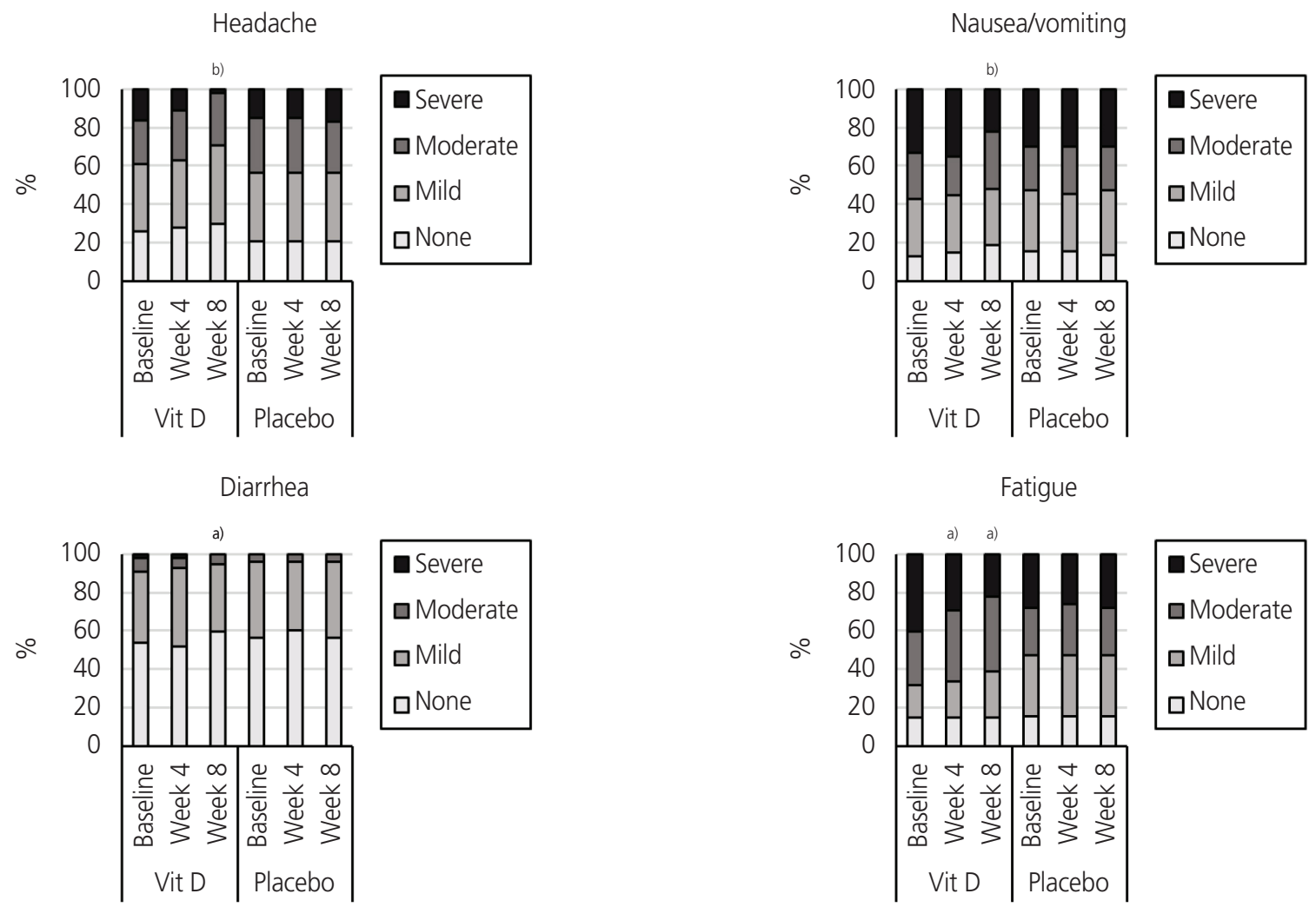

Fig. 3. Systemic symptoms severity over the study period in the vitamin $D$ and placebo groups. ${ }^{\text {a) }} P<0.05$; ${ }^{\text {b) }} P<0.001$. 


\title{
Obstetrics \& Gynecology Science
}

\author{
Vol. 64, No. 4, 2021
}

low serum calcium levels can cause uterine smooth muscle contraction and spasm [15]. Additionally, the widespread presence of vitamin $D$ receptors throughout the human body and the expression of 1-a hydroxylase in myometrial cells containing vitamin $D$ suggests the possible role of vitamin $D$ in the pathogenesis of primary dysmenorrhea and its associated systemic symptoms $[19,33,34]$. Therefore, vitamin $D$ deficiency may attribute to the pathogenesis of primary dysmenorrhea or to the severity of symptoms.

Vitamin D metabolites reduce the production of prostaglandin in the uterine endometrium and restrict its biological activity by affecting prostaglandin receptors in the endometrium [18]. Lasco et al. [21] used a single 300,000 IU dose of vitamin $\mathrm{D}$ five days before menstruation in the two menstrual cycles in women with painful menstruation who also had a serum vitamin D level of $<45 \mathrm{ng} / \mathrm{mL}$ (low quartile of the community) and observed a reduction in dysmenorrhea symptoms following the treatment. In a similar clinical trial by Zangene et al. [22], a single 300,000 IU dose of vitamin D, five days before menstruation, was found effective and safe in women with primary dysmenorrhea. We used a weekly dose of 50,000 IU vitamin D for 8 weeks according to the Endocrine Society clinical practice guideline [26]. The method of vitamin $D$ administration in our study was similar to that of Moini et al's [23] study. They also found a reduction in pain severity and frequency and the number of painkillers consumed by women with primary dysmenorrhea following a weekly dose of 50,000 IU vitamin D for 8 weeks. According to the available studies, vitamin D supplementation, both in single and multiple doses, can reduce the severity of primary dysmenorrhea. Available guidelines recommend that the maximum permitted dose of vitamin $D$ supplements for young adults is 6,000-10,000 IU per day [26]. In the present study, the dose of vitamin $D$ used was within the safe range and less likely to trigger the occurrence of vitamin $D$ toxicity and complications [35].

Studies using high weekly doses of vitamin D (e.g., 30,000 to $50,000 \mathrm{IU} /$ week) have reported a significant increase in serum vitamin D levels after 4 weeks $[36,37]$. In fact, higher doses of vitamin $D$ can result in the speedy correction of vitamin $\mathrm{D}$ deficiency, but it does not raise safety concern according to previous review studies [38]. According to our data, the improvement in pain severity may already be visible 4 weeks after supplementation with high-dose vitamin D, and sustained improvement can be observed with continu- ous supplementation up to 8 weeks. It remains unclear whether continuing supplementation beyond 8 weeks could further improve the symptoms, and this requires further studies with longer duration and follow-ups. In our study, complete correction of vitamin D level was achieved in 75\% of the participants who received vitamin D supplements, but $25 \%$ still had low levels $(<30 \mathrm{ng} / \mathrm{mL}$ ) after 8 weeks. However, the levels were higher than $20 \mathrm{ng} / \mathrm{mL}$ in participants with vitamin D insufficiency [26]. Whether these participants could experience more relief in symptoms by continuing vitamin $D$ supplementation until complete vitamin D level correction is achieved remains unknown. No significant correlations were found between the increase in vitamin D level and improvement in pain; as regards pain intensity, no significant difference was observed between participants who achieved normal vitamin D level $(\geq 30 \mathrm{ng} / \mathrm{mL})$ and those who did not at week 8 . These results suggested that vitamin $D$ supplementation (50,000 IU/week for 8 weeks) could improve the symptoms regardless of whether vitamin $D$ deficiency is completely corrected or not, and that the degree of improvement is not linearly correlated with the degree of change in serum vitamin $\mathrm{D}$ level. The mechanisms by which vitamin $\mathrm{D}$ supplementation can reduce the pain in patients with primary dysmenorrhea is not known. Hence, future studies must measure the prostaglandins and other potential mediators during supplementation in order to investigate the aforementioned mechanisms.

The severity of systemic symptoms associated with primary dysmenorrhea (headache, fatigue, nausea, vomiting, and diarrhea) improved in general after treatment with vitamin $D$ in our study. This could be attributed to the possible role of prostaglandins in the pathogenesis of the systemic symptoms that accompany pain in dysmenorrhea [2]. Karacin et al. [39] examined 12 physical and psychological symptoms in women with primary dysmenorrhea and found a negative association between the severity of the symptoms and the levels of vitamin D. In the investigations related to premenstrual syndrome, the symptoms such as physical pain, fatigue, vomiting, nausea, and diarrhea were assessed; it was found that calcium deficiency was associated with the severity of the symptoms [16]. It could be assumed that vitamin D and parathyroid hormone play a role in the intensity of systemic physical and mental symptoms of premenstrual syndrome [40]. Moreover, taking calcium and vitamin D supplements will improve the physical and psychological symptoms of pre- 


\section{Obstetrics \& Gynecology Science}

Fatemeh Alsadat Rahnemaei, et al. Vitamin D for primary dysmenorrhea

menstrual syndrome and primary dysmenorrhea $[16,17]$. We did not measure the calcium level in this study, and its role in the development of symptoms and responses to vitamin D supplementation in patients with primary dysmenorrhea warrants further investigations.

This study had certain limitations. Since the study was conducted among the students residing in the university dormitories, our findings cannot be generalized to the general population. Given that the participants with a serum level of $<30 \mathrm{ng} / \mathrm{mL}$ (vitamin D deficiency) were enrolled in our study, it is not possible to comment on the effect of vitamin D supplementation on the severity of primary dysmenorrhea in people with normal vitamin D levels. Further research is needed to determine the cut-off value for vitamin D levels contributing to the pathophysiology of primary dysmenorrhea and its associated systemic symptoms, as well as investigate the effect of vitamin D supplements on the improvement of primary dysmenorrhea in people with normal vitamin D levels.

In summary, this study shed light to the fact that vitamin D supplementation in women with primary dysmenorrhea and vitamin D deficiency can improve systemic symptoms and reduce the pain intensity, the number of days with pain, and the need for consuming pain-relief medications. The use of vitamin D supplements could be an effective and safe approach for the complementary treatment of primary dysmenorrhea.

\section{Conflict of interest}

No potential conflict of interest relevant to this article was reported.

\section{Ethical approval}

Necessary permissions were obtained from the Ethics Committee of the Faculty of Nursing and Midwifery at Shahid Beheshti University of Medical Sciences (IR.SBMU. PHNM.1396.772). The trial was registered at the Iranian Registry of Clinical Trials (code no. IRCT201707223860N32). The study was performed in accordance with the principles of the Declaration of Helsinki.

\section{Patient consent}

Written informed consent and the use of images from patients are not required for the publication.

\section{Funding information}

None.

\section{Acknowledgments}

This article is based on an MSc thesis. We acknowledge the contribution of the research committee of students, Faculty of Nursing and Midwifery and Deputy Director of Research and Technology of Shahid Beheshti University of Medical Sciences, dormitories department, and staff of Shohadaye Tajrish laboratory to this research.

\section{References}

1. ACOG Committee Opinion No. 760: Dysmenorrhea and endometriosis in the adolescent. Obstet Gynecol 2018;132:e249-58.

2. lacovides S, Avidon I, Baker FC. What we know about primary dysmenorrhea today: a critical review. Hum Reprod Update 2015;21:762-78.

3. Proctor $M$, Farquhar C. Diagnosis and management of dysmenorrhoea. BMJ 2006;332:1134-8.

4. Juang CM, Yen MS, Twu NF, Horng HC, Yu HC, Chen CY. Impact of pregnancy on primary dysmenorrhea. Int J Gynaecol Obstet 2006;92:221-7.

5. Ju H, Jones M, Mishra $G$. The prevalence and risk factors of dysmenorrhea. Epidemiol Rev 2014;36:104-13.

6. Kharaghani R, Damghanian M. The prevalence of dysmenorrhea in Iran: a systematic review and meta-analysis. Iran Red Crescent Med J 2017;19:e40856.

7. De Sanctis V, Soliman AT, Elsedfy H, Soliman NA, Elalaily $R$, El Kholy M. Dysmenorrhea in adolescents and young adults: a review in different countries. Acta Biomed 2016;87:233-46.

8. Sultan C, Gaspari L, Paris F. Adolescent dysmenorrhea. Endocr Dev 2012;22:171-80. 


\section{Obstetrics \& Gynecology Science}

Vol. 64, No. 4, 2021

9. Doty E, Attaran M. Managing primary dysmenorrhea. J Pediatr Adolesc Gynecol 2006;19:341-4.

10. Berek JS. Berek \& Novak's gynecology. 16th ed. Philadelphia (PA): Lippincott Williams \& Wilkins; 2019.

11. Guimarães I, Póvoa AM. Primary dysmenorrhea: assessment and treatment. Rev Bras Ginecol Obstet 2020;42:501-7.

12. Zahradnik HP, Hanjalic-Beck A, Groth K. Nonsteroidal anti-inflammatory drugs and hormonal contraceptives for pain relief from dysmenorrhea: a review. Contraception 2010;81:185-96.

13. Firestein GS, Budd RC, Harris T, Maclnnes IB, Ruddy S, Sergent JS. Kelley's textbook of rheumatology. 8th ed. Philadelphia (PA): Saunders Elsevier; 2009.

14. Pramanik P, Banerjee SB, Saha P. Primary dysmenorrhea in school going adolescent girls-is it related to deficiency of antioxidant in diet? Int J Life Sci Phar Res 2015;5:5463.

15. Charandabi SMA, Mirghafourvand $M$, Javadzadeh $Y$, Chegini SN. Effect of calcium with and without magnesium on amount and duration of menstrual bleeding in students with primary dysmenorrhea. Iran J Obestet Gynecol Infertil 2013;16:1-8.

16. Abdi F, Ozgoli G, Rahnemaie FS. A systematic review of the role of vitamin $D$ and calcium in premenstrual syndrome. Obstet Gynecol Sci 2019;62:73-86.

17. Abdi F, Amjadi MA, Zaheri F, Rahnemaei FA. Role of vitamin $D$ and calcium in the relief of primary dysmenorrhea: a systematic review. Obstet Gynecol Sci 2021;64:13-26.

18. Thota C, Laknaur A, Farmer T, Ladson G, Al-Hendy A, Ismail $N$. Vitamin $D$ regulates contractile profile in human uterine myometrial cells via NF-kB pathway. Am J Obstet Gynecol 2014;210:347.e1-10.

19. Viganò $P$, Lattuada $D$, Mangioni $S$, Ermellino L, Vignali $M$, Caporizzo $E$, et al. Cycling and early pregnant endometrium as a site of regulated expression of the vitamin $D$ system. J Mol Endocrinol 2006;36:415-24.

20. Kucukceran H, Ozdemir O, Kiral S, Berker DS, Kahveci R, Ozkara A, et al. The impact of circulating 25-hydroxyvitamin and oral cholecalciferol treatment on menstrual pain in dysmenorrheic patients. Gynecol Endocrinol 2019;35:53-7.

21. Lasco A, Catalano A, Benvenga S. Improvement of primary dysmenorrhea caused by a single oral dose of vita- min D: results of a randomized, double-blind, placebocontrolled study. Arch Intern Med 2012;172:366-7.

22. Zangene $M$, Veisi F, Nankali A, Rezaei $M$, Ataee $M$. Evaluation of the effects of oral vitamin-D for pelvic pain reduction in primary dysmenorrhea. Iran J Obstet Gynecol Infertil 2014;88:14-20.

23. Moini A, Ebrahimi T, Shirzad N, Hosseini R, Radfar M, Bandarian $F$, et al. The effect of vitamin $D$ on primary dysmenorrhea with vitamin $D$ deficiency: a randomized double-blind controlled clinical trial. Gynecol Endocrinol 2016;32:502-5.

24. Ayşegül Ö, Seda A, Şevket O, Özdemir M, Illhan G, Davutoğlu $\mathrm{E}$. A randomized controlled study of vitamin $D$ in the treatment of primary dysmenorrhea. Duzce Med J 2019;21:32-6.

25. Vatandost S, Jahani M, Afshari A, Amiri MR, Heidarimoghadam $R$, Mohammadi $Y$. Prevalence of vitamin $D$ deficiency in Iran: a systematic review and meta-analysis. Nutr Health 2018;24:269-78.

26. Holick MF, Binkley NC, Bischoff-Ferrari HA, Gordon CM, Hanley DA, Heaney RP, et al. Evaluation, treatment, and prevention of vitamin $\mathrm{D}$ deficiency: an Endocrine Society clinical practice guideline. J Clin Endocrinol Metab 2011;96:1911-30.

27. Beller EM, Gebski V, Keech AC. Randomisation in clinical trials. Med J Aust 2002;177:565-7.

28. Hodges JL Jr, Lehmann EL. Estimates of location based on rank tests. Ann Math Stat 1963;34:598-611.

29. Holm S. A simple sequentially rejective multiple test procedure. Scand J Statist 1979;6:65-70.

30. Abdul-Razzak KK, Ayoub NM, Abu-Taleb AA, Obeidat $B A$. Influence of dietary intake of dairy products on dysmenorrhea. J Obstet Gynaecol Res 2010;36:377-83.

31. Abdul-Razzak KK, Obeidat BA, Al-Farras MI, Dauod AS. Vitamin D and PTH status among adolescent and young females with severe dysmenorrhea. J Pediatr Adolesc Gynecol 2014;27:78-82.

32. Holick MF. Vitamin D deficiency. N Engl J Med 2007;357:266-81.

33. Adams JS, Hewison M. Extrarenal expression of the 25-hydroxyvitamin D-1-hydroxylase. Arch Biochem Biophys 2012;523:95-102.

34. Abbasi ST, Abbasi P, Suhag AH, Qureshi MA. Serum magnesium and 25-hydroxy cholecalciferol in premenstrual syndrome during luteal phase. J Liaquat Uni Med 


\section{Obstetrics \& Gynecology Science}

Fatemeh Alsadat Rahnemaei, et al. Vitamin D for primary dysmenorrhea

Health Sci 2017;16:209-12.

35. Holick MF, Binkley NC, Bischoff-Ferrari HA, Gordon CM, Hanley DA, Heaney RP, et al. Guidelines for preventing and treating vitamin $D$ deficiency and insufficiency revisited. J Clin Endocrinol Metab 2012;97:1153-8.

36. Fassio A, Adami G, Rossini M, Giollo A, Caimmi C, Bixio $R$, et al. Pharmacokinetics of oral cholecalciferol in healthy subjects with vitamin d deficiency: a randomized open-label study. Nutrients 2020;12:1553.

37. Toth BE, Takacs I, Szekeres L, Szabo B, Bakos B. Safety and efficacy of weekly 30,000 IU vitamin D supplementation as a slower loading dose administration compared to a daily maintenance schedule in deficient patients: a randomized. Contr Clin Trial J Pharmacovigil 2017;5:2.

38. Kearns MD, Alvarez JA, Tangpricha V. Large, single-dose, oral vitamin D supplementation in adult populations: a systematic review. Endocr Pract 2014;20:341-51.

39. Karacin O, Mutlu I, Kose M, Celik F, Kanat-Pektas M, Yilmazer M. Serum vitamin D concentrations in young Turkish women with primary dysmenorrhea: a randomized controlled study. Taiwan J Obstet Gynecol 2018;57:58-63.

40. Thys-Jacobs S, McMahon D, Bilezikian JP. Cyclical changes in calcium metabolism across the menstrual cycle in women with premenstrual dysphoric disorder. J Clin Endocrinol Metab 2007;92:2952-9. 